\title{
PRECISION DETERMINATION OF FOCAL DEPTHS AND EPICENTERS OF LOCAL SHOCKS IN CALIFORNIA
}

\author{
By Armando Cisternas
}

\begin{abstract}
A program for the location of hypocenters of local shocks by means of an electronic digital computer is described. The $P$ times are corrected for variations in crustal structure to get precision in the determination of depth. An estimate of the accuracy of the method is given for some particular cases. Application of the program to the Kern County series of aftershocks, provides a clearer picture of tectonic mechanism of the White Wolf fault. A future extension of the program to process and correct data automatically is also described.
\end{abstract}

\section{INTRODUCTION}

The problem of precision location of the hypocenter of earthquakes is becoming increasingly important. It is greatly simplified by the use of electronic computers, which make it possible to undertake rapid computation of large amounts of data.

Some results which might be obtained with computer techniques are:

(a) Accurate determination of epicenter and depth of earthquakes, and elimination of some earthquakes from suspicion as explosions, by using focal depths as criteria.

(b) More insight in the study of the earthquake mechanism by providing the distribution of the aftershocks in three dimensions.

(c) Construction of accurate travel-time curves for regions with uniform crust, when sufficient direct arrival data are available to determine precise hypocenters. This requires several stations (possibly temporary ones) within 150 kilometers of the epicenter, as indicated in figure 1.

(d) Investigation of crustal structure in rugged regions, by interpretation of systematic deviations of travel time data.

In this paper we present an initial study of these problems for focal shocks in California, using the first arrivals of $P$ waves as input data. A more sophisticated program will also include other crustal phases.

\section{Description of the Program}

In the Caltech Seismological Laboratory at Pasadena there are two programs which provide routine determinations of hypocenters; one written by Mr. John M. Nordquist is designed specifically for local shocks (Nordquist, 1962) and the other, written by Mr. Saul Shragowitz for teleseisms, has been adapted by the author to compute local shocks as well. Both of these use the Bendix G-15 computer.

In the program for distant shocks, accurate direction cosines were included for the local network of stations, and travel time tables were calculated from Press' model for the California-Nevada region (Press, 1960). This model consists of a two layer crust with compressional velocities 6.11 kilometers/second and 7.66 kilometers/second, and 8.11 kilometers/second for the mantle. The thicknesses are 25 and 26 kilometers respectively. Another interpretation of crustal structure which fits Press' travel time data would not materially change our results. 
By using the method of least squares (Byerly, 1942, p. 217) an initial trial for the focus is changed in such a way as to minimize the deviations of the actual times to the stations, from the ones given in the tables. These deviations will be called "residuals".

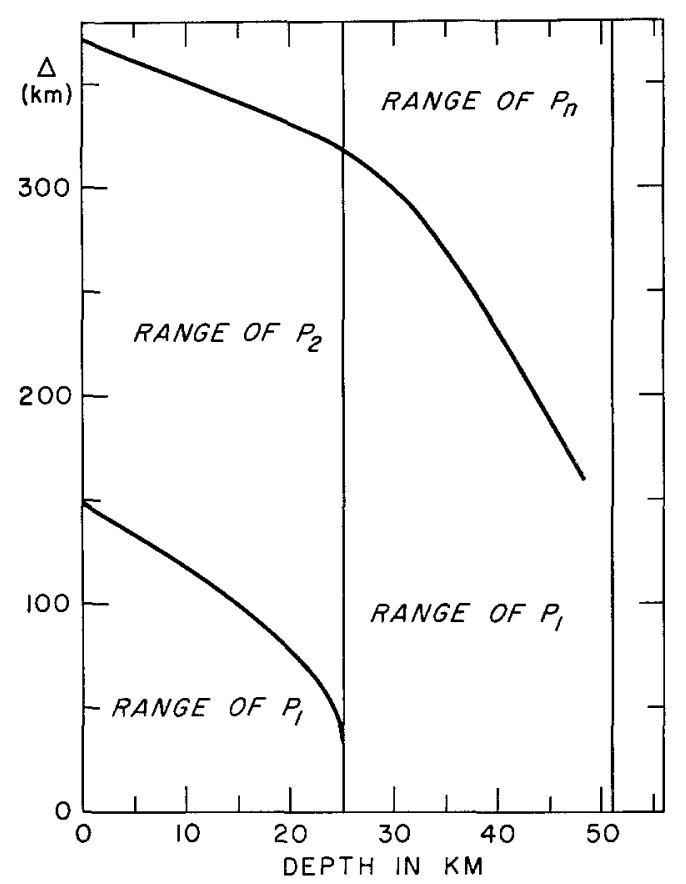

FIg. 1. First arrival as a function of distance and depth of the focus, for the crustal model used in the computer program.

The solution of the normal equations can be written in matrix notation (Bolt, 1960):

$$
\left[\begin{array}{c}
\Delta \lambda \\
\Delta \varphi \\
\Delta h \\
\Delta t_{0}
\end{array}\right]=\left[\begin{array}{llll}
A_{1} & A_{2} & \cdots & A_{n} \\
B_{1} & B_{2} & \cdots & B_{n} \\
C_{1} & C_{2} & \cdots & C_{n} \\
D_{1} & D_{2} & \cdots & D_{n}
\end{array}\right] \times\left[\begin{array}{c}
\delta t_{1} \\
\delta t_{2} \\
\vdots \\
\delta t_{n}
\end{array}\right]
$$

where $\Delta \lambda, \Delta \varphi, \Delta h, \Delta t_{0}$, are the changes in longitude, latitude depth and origin time, to be made in the guess. The large matrix is a function of the slopes of the travel time curves at the several distances between the epicenter and each one of the stations, $\delta t_{i}$ is the residual at the $i$ th station.

From (1) we see that if the travel time tables are linear, one iteration is sufficient, since the matrix depends on the slopes, which are constants. The actual tables do not deviate much from linearity except at the origin, and we can expect a fast convergence if the data are consistent. From equation (1) we can also compute the effect of a time error at any station on the solution. 
The input to the program consists of code numbers that identify the stations, the arrival time for each station, and an initial guess for the hypocenter.

The output after each iteration is printed out in the following pattern:

Code; $\Delta_{i}(\mathrm{~km}) ;\left(t_{0}\right)_{i} ; P i-\Delta_{i} / 8.11 ;$ (Residual) $_{i}$.

Following this, the machine types out the standard deviation in time, $\sigma=(1 /[n] \Sigma$ $\left.(\text { Residuals })^{2}\right)^{1 / 2}$, and then it prints the next trial:

$$
\lambda^{\circ} ; \varphi^{\circ} ; h(\mathrm{~km} .) ; t_{0}
$$

The iterations continue unless the machine is stopped manually. If 15 stations are processed, the time per iteration is 7.5 minutes. (Programming is now underway for a large computer which among other things will greatly shorten this time.)

The program gives focal depths smaller than 56 kilometers only, which is sufficient for all the cases known in California.

\section{The Merhod: Its Assumptions and Sources of Errors}

The method is based on the precise location of a series of shocks in a local region where a relatively dense (and temporary) network of stations was available. Systematic deviations in travel times to more distant (permanent) stations were obtained. These deviations were then used to obtain station corrections which made possible precision determinations of hypocenters in the same general region but with fewer stations. The station corrections were then interpreted in terms of crustal structure variations along the path connecting the stations and the source.

For simplicity the travel time tables were computed from a two-layer model of the crust, with constant velocities and constant thicknesses throughout the region. The layers were considered plane, horizontal, and isotropic. Nevertheless, the curvature of the earth is not negligible for distances between 300 and 1000 kilometer. The difference between horizontal distances measured at the surface of the earth and at the Mohorovičic discontinuity is $\Delta-\Delta^{1} \approx H \Delta / R$, where $H$ is the thickness of the crust and $R$ the radius of the earth. For $\Delta=1000$ kilometer and $H=40$ kilometer, $\Delta-\Delta^{1} \approx 7$ kilometer, which corresponds to a time error of 0.8 seconds in a $P_{n}$ arrival. This amount is large and a correction should be made for epicentral distances greater than some 200 kilometer. This was not necessary for our studies of the Kern County aftershocks.

The effect of ellipticity is too small to be considered in any case. We used the radius of the mean sphere to compute distances along great circles. To estimate the error so produced, we may consider that the distance along a meridian is given by $\Delta^{1} \approx a[1-(\delta \cos \varphi) /(\delta \varphi) \cdot e] \delta \varphi$, to the first order in the ellipticity $e$ ( $a$ is the radius at the equator, $\varphi$ the latitude). The distance computed by the program is $\Delta=a\left(1-\frac{2}{3} e\right) \delta \varphi$ if we take the end points at $32^{\circ}$ and $42^{\circ}$, the difference between the two calculations is $\Delta-\Delta^{1} \approx 0.12$ kilometer.

No consideration was given to lateral refraction, or other variations along the path, due to our lack of knowledge of the local structure. Also corrections due to the sedimentary layers were unnecessary since all of the stations are located on bedrock. There are indications of lower velocities in the upper layers of the crust, but we believe that the average values used in our computations give focal depths which are not likely to change by more than a few kilometers in depth. 
These sources of errors will not seriously affect the validity of results obtained for epicenters located in a restricted region (for example, the shocks around the White Wolf fault). This follows because small changes in epicenters and depth do not materially change the propagation paths. For this case deviation in travel times from the assumed model may be used to obtain station corrections which make the real earth consistent with the model. For the more general case of widely distributed epicenters, the preceding sources of error cannot be neglected.

The accuracy and stability of the solutions are very difficult to test theoretically. However, it is rather easy to check some specific examples. This we did in three ways: (a) By direct comparison with the data of explosions. The use of the program for the Corona and Victorville explosions (Press, 1960) gave error in depth of 1 kilometer and 3 kilometer respectively. The epicenters were off by 1.5 kilometer and 2.7 kilometer. (b) By selecting well recorded earthquakes (with seven or more stations within the range of the direct wave) and comparing the very accurate hypocenters thus determined, with the ones obtained using raw data from

TABLE 1

\begin{tabular}{|c|c|c|c|c|}
\hline $\begin{array}{l}\text { No. of shock } \\
\text { according to Richter } \\
(1955)\end{array}$ & Mag. & $\Delta(\mathrm{km})$ & $\Delta(\mathrm{km})$ & $\Delta h(\mathrm{~km})$ \\
\hline 285 & 5.1 & -8 & -8 & 34 \\
\hline 155 & 5.8 & -6.7 & -8.5 & 68 \\
\hline 276 & 5.9 & -6.7 & -11.4 & 24 \\
\hline
\end{tabular}

An example of the errors involved when no crustal corrections are applied and the direct wave is not included. (See paragraph $3 \mathrm{~b}$ ).

distant stations only. This error estimate can be attributed to crustal variations and shows how important it is to correct for these variations if precision focal depths are desired. Some examples of this effect are shown in table 1. (c) By studying the change in focus, when a unit change was made on the arrival time of each of the stations, in such a sense (plus or minus) as to produce the maximum deviation in the corrections $\Delta \lambda, \Delta \varphi, \Delta h$, or $\Delta t_{0}$. For the main Kern County shock, with a net of 12 stations, and a variation of .1 seconds in each of them we got the following result: $d h=1.28$ kilometer, $d \lambda=2.7$ kilometer, $d \varphi=1.1$ kilometer.

The geometry of the distribution of the stations around the epicenter also plays an important role upon the confidence we may have in a given determination. It is obvious that the best epicentral location will be that in which the stations are evenly distributed in azimuth. But we can also get good control with sufficient near stations, even if the distribution is not optimal.

As Nordquist (1962) pointed out, the precision in depth will depend on the type of wave recorded. For instance, if we get only $P_{2}$ arrivals there is no control on depth, because a variation in depth will be compensated by a shift in origin time. If $P_{2}$ and $P_{n}$ are present, there is control but very little. The depth can be tied down with more certainty only when the direct wave is present. In any case, due to the properties of the rays, the uncertainty is not complete. The $P_{2}$ arrival is first within a range which depends upon the depth (see figure 1). For a fixed distance, an 
increase in the focal depth will allow the direct wave to arrive first. Similar considerations hold for $P_{n}$ and $P_{1}$.

\section{Application to the Kern County Series of Aftershocks}

The Kern County series of aftershocks (starting July 21, 1952) occurred in a limited area, and the ray paths to the several stations can be considered almost constant. Moreover, the number of portable stations installed in the epicentral region was enough to locate a set of some 25 aftershocks of magnitude over 4 , using data from the direct waves only.

The great majority of the aftershocks were located close to the edges of the White Wolf fault. We classified them in three groups according to their location. Group A includes those on the SW end of the fault, Group C those on the NE corner and Group B those in between.

With accurate origin times of aftershocks based on data from near stations, and the arrival times to the distant stations we were able to determine the actual travel times for each path. The differences from the travel times for our assumed model, were interpreted as being due to variations in crustal structure. These differences were also used as corrections to the arrival times of the corresponding stations for other shocks which were not so widely recorded. By applying such corrections we succeeded in getting precision depths for most of the aftershocks where at least one station received the direct wave. Figure 2 shows the distribution of the aftershocks in a horizontal plane, the sense of motion is according to Richter and Bath (1958), and figure 3 shows our depth distributions of the aftershocks in a projection over a plane perpendicular to the strike of the White Wolf fault plane.

Observation of figure 3 shows that the tectonic activity was mostly confined to the northwestern side of the White Wolf fault. We call this region the "active block". Almost all of the aftershocks in figure 2 that are on the southeastern side of the trace of the fault, lay very close to its assumed plane, when projected downward to the depths we have determined. The general trend of the motion of the "active blocks" is upward and toward the SW, as follows from the sequence of aftershocks worked out by Bath and Richter (1958), on the basis of first motion $P$ waves. In figure 2 there are 29 shocks having a left-hand strike-slip motion, against 9 with a right-hand motion.

Geodetic measurements (Whitten, 1955, p. 79) give relative displacements which agree with the ones above, in the horizontal component, but not in the vertical. Moreover the main shock had the characteristic of an overthrust motion (Gutenberg, 1955). It is not clear how to pass from the overthrust dip-slip motion of the main shock to the mechanism of the strike-slip and normal dip-slip aftershocks. A theory based on a rebound effect would explain only the differences in vertical motion.

In contrast with the dominant left-hand strike-slip motion, the shocks 194, 141 and adjacent ones in figure 2 have right-hand strike-slip motion. Båth and Richter conclude that they correspond to a secondary release of energy far from the main fault, and they wonder why it is not present on the southern side too. It is clear now that we should not expect this, since our new hypocenters indicate that this showed little seismic activity.

The distribution of the aftershocks within the active block is such that in the 
projection shown in figure 3 it is easy to observe linear trends. This suggests the possibility of several major breaks rather than one. Some tentative location for the breaks are indicated in figure 3 by dashed lines.

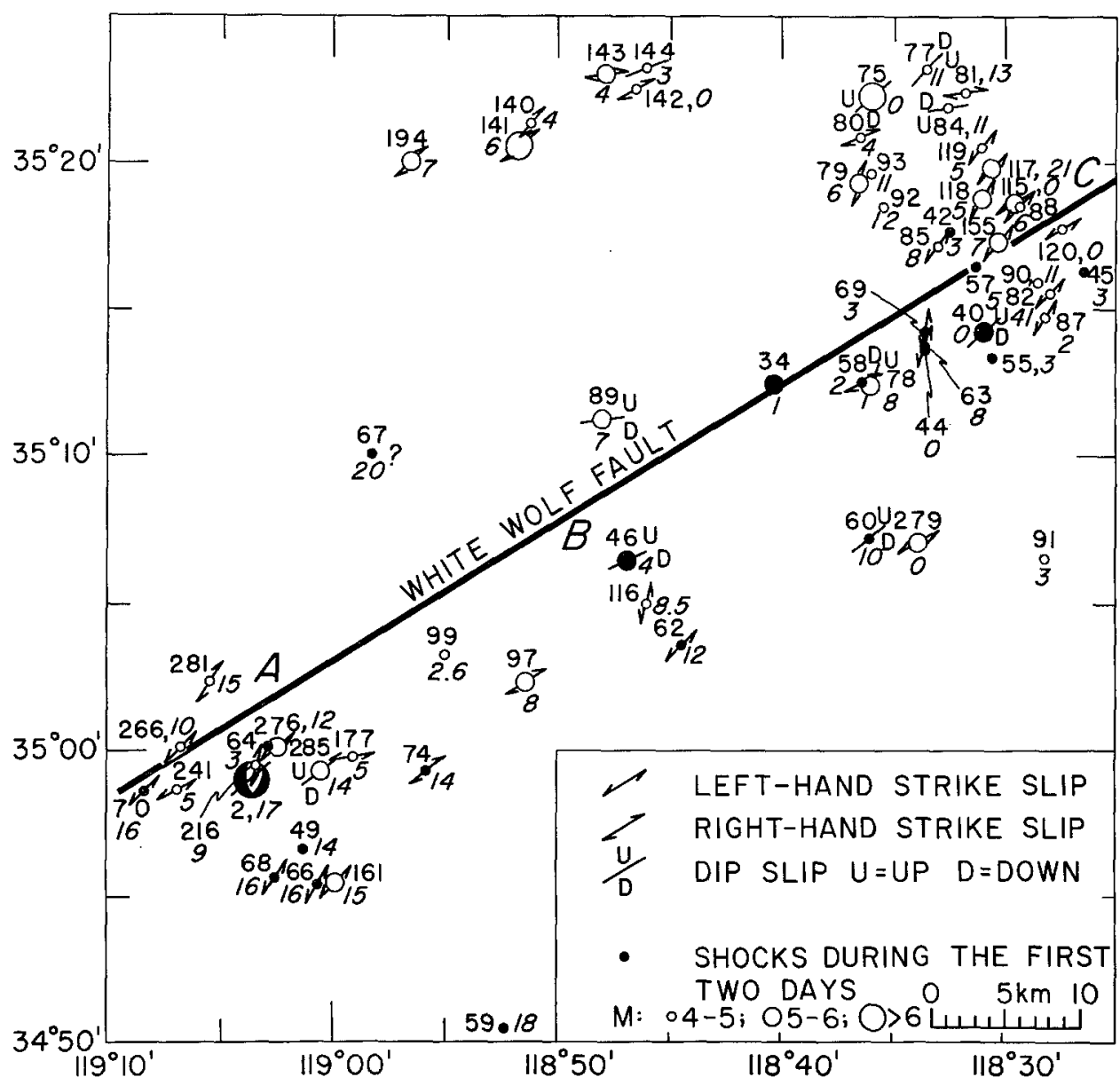

FIG. 2. Horizontal distribution of the epicenters of the Kern County aftershocks. The sense of motion is taken from Bâth and Richter (1958). The figures on the upper part of the circles refer to the classification of the aftershocks according to Richter (1955), the lower ones are the depths in $\mathrm{km}$.

\section{Interpretation of the Corrections in Terms of Regional Variations in Crust}

To interpret physically the deviations from the model from which we computed the travel times, we must account for horizontal variations in the velocities of the layers, the depths of the discontinuities and lateral refraction.

A list of the residuals at the several stations for shocks of type $A$ and $C$ are given in table 2.

Assuming that the velocities do not change horizontally, we can interpret the 
residuals at Tinemaha and Haiwee as a deepening of the Conrad discontinuity by some 5 kilometers under the Sierra Nevada. This increase should be associated with the offset point where the ray begins to emerge. A similar calculation for the residuals

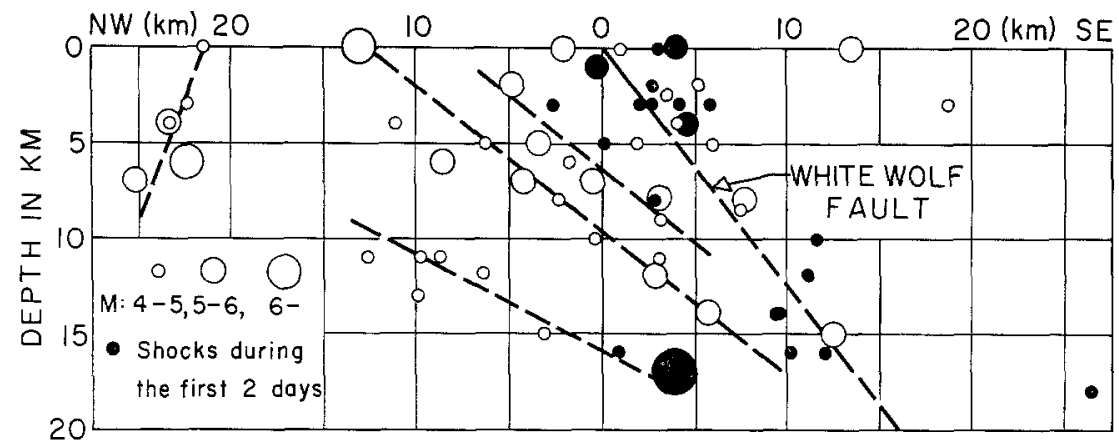

Fig. 3. Vertical cross section showing the distribution of the aftershocks in depth. The dashed lines indicate possible breaks north of the main fault.

TABLE 2

\begin{tabular}{|c|c|c|c|c|}
\hline \multirow{2}{*}{ Station } & \multicolumn{2}{|c|}{$\begin{array}{c}\text { From } \\
\text { Region A }\end{array}$} & \multicolumn{2}{|c|}{$\begin{array}{c}\text { From } \\
\text { Region C }\end{array}$} \\
\hline & Residual sec. & Standard dev. sec. & Residual sec. & Standard dev. sec. \\
\hline Pasadena............. & 0.01 & & -0.07 & 0.11 \\
\hline Mt. Wilson. . . . . ...... & 0.03 & 0.21 & 0.14 & 0.26 \\
\hline Dalton ............... & 0.11 & 0.31 & -0.02 & 0.35 \\
\hline Riverside............ & 0.29 & 0.22 & -0.02 & 0.36 \\
\hline Big Bear.............. & 0.22 & 0.31 & 0.02 & 0.24 \\
\hline Sta. Barbara .......... & 0.02 & 0.16 & 1.53 & 0.18 \\
\hline China Lake . . . . . . . . . & 0.76 & 0.19 & -0.15 & 0.16 \\
\hline Haiwee............... & 1.59 & 0.29 & 0.27 & 0.17 \\
\hline Tinemaha........... & 1.67 & 0.32 & 1.76 & 0.27 \\
\hline Fresno . ............... & -0.38 & 0.35 & - & - \\
\hline Palomar.............. & -0.29 & 0.12 & -0.43 & 0.28 \\
\hline Barrett............... & -0.63 & 0.28 & -0.47 & 0.22 \\
\hline Berkeley . ............ & -1.47 & 0.54 & - & - \\
\hline Mt. Hamilton . . . & -1.75 & 0.34 & - & - \\
\hline
\end{tabular}

Time deviations from the model with horizontal layering, due to crustal variations, for shocks in the Kern County area. Regions A and C refer to figure 2.

at Berkeley, San Francisco, and Mount Hamilton suggests that the crust is some 8 kilometers thinner at these points (thickness to the Moho). In the arrivals to Barrett a residual of $-0.6 \mathrm{sec}$ is interpreted as a 4 kilometers thinner crust.

For shocks in the C location the residuals at Santa Barbara are high; this effect could be due to the presence of the upper tertiary and quaternary sediments in the region (Reed and Hollister, 1936).

\section{SECONd ORder CoRrections}

We obtained the previous results by the introduction of a crustal correction 
$\Delta t_{i}$ to the travel time of the $i$ th station. The new hypocenters gave a set of residuals that were very small, but still not zero. A further step was taken to improve the correction $\Delta t_{i}$, adding to it a second order parameter $\xi_{i}$ in such a way as to minimize the sum of the squares of the residuals for all the processed shocks. It was found that the second order corrections $\xi_{i}$, were given by the average sum of the residuals of all the shocks at the $i$ th station.

The numerical values of the $\xi_{i}$ were small, the largest being of the order of .1 second. Our confidence in the analysis was thus confirmed, and we therefore concluded that no further changes in the station corrections were necessary.

\section{Extension of the Method to Any Local Shock}

In this paper we have described a method for improved hypocenter location for earthquakes in a local region, involving empirical station corrections to the travel times. To extend these results for the more general case a new program will be needed, which will include corrections for structural changes along the entire path. At first these corrections will not be directly available to us, and we will need to deduce them from reasonable assumptions relating crustal structure to topography and gravity anomaly. Velocities will be allowed to vary along the path. As the California region is not homogeneous we will establish different crustal parameters for each region considered as a Geologic Province. Corrections for sediments, when needed, will be included.

As soon as more reliable data on crustal structure is available from direct measurements, they will replace the values deduced indirectly.

The complexity of this program will require the use of a computer in the IBM 7090 class.

\section{Conclusions}

The determination of epicenters and depths of local shocks in regions as rugged as California, can be made with an accurace of 2 to 3 kilometers by carefully correcting the travel times to account for major sources of errors. One of the most important corrections is for variations in crustal structure. This correction can be obtained if a sufficiently dense network of stations is available as is the case in California.

The crustal corrections computed in California are consistent with crustal thickness variations as indicated by gravity and topography.

The Kern County series of aftershocks of 1952, occurred mostly in one active block, and the depth distribution provided in this paper is in agreement with the sense of motion given by Båth and Richter (1958).

\section{ACKNowledgMeNTS}

This paper represents research conducted under Grant No. AF-AFOSR-25-63 of the Air Force Technical Applications Center as part of the Advanced Research Projects Agency's Project vela uniform. The author wishes to thank Dr. Frank Press and Dr. Charles Richter for valuable suggestions and comments.

\section{References}

Båth, M., and C. F. Richter

1958. "Mechanism of the Aftershocks of the Kern County, California Earthquake of 1952," Bull. Seism. Soc. Am. 48, 133-146. 
Byerly, P.

1942. Seismology. Prentice-Hall, N. Y.

Bolt, B. A.

1960. "Earthquake Epicenters, Focal Depths and Origin Times Using a High-Speed Computer," Geophys. Jour. 3, 433-440.

Gutenberg, B.

1955. "The First Motion in Longitudinal and Transverse Waves of the Main Shock and the Direction of Slip," Bull. Div. of Mines, No. 171, 165-170.

Nordquist, J. M.

1962. "A Special Purpose Program for Earthquake Location With An Electronic Computer," Bull. Seism. Soc. Am. 52, 431-437.

Press, F.

1960. "Crustal Structure in the California-Nevada Region," Jour. Geophys. Res., 65, 1039-1051.

Richter, C. F.

1955. "Foreshocks and Aftershocks," Bull. Div. of Mines, No. 171, 177-197.

Reed, R. D.

1936. "Structural Evolution of Southern California," AAPG. Tulsa, Okla.

Scheidegger, A. E.

1959. "Note on the Tectonics of Kern County, California, As Evidenced by the 1952 Earthquakes," Jour. Geophys. Res. 61, 1499-1501.

Whitten, C. A.

1955. "Measurement of Earth Movements in California," Bull. Div. of Mines, No. 171. $75-80$.

Stismological Laboratory

California Institute of Technology

Pasadena, California

(Division of the Geological Sciences, Contribution No. 1142)

Manuscript received February 26, 1963. 